\title{
Chemical Composition and Antibacterial Activity of the Essential Oil from Qiancao (Rubia cordifolia Linn.) Roots against Selected Foodborne Pathogens
}

\author{
Wei-Qin $\mathrm{Li}^{1 *}$, Mei-Ping Quan ${ }^{2}$, Qin $\mathrm{Li}^{1}$ \\ ${ }^{1}$ School of Food Sciences, Shanxi Normal University, Linfen, China. \\ ${ }^{2}$ College of Chemistry and Life Science, Weinan Normal University, Weinan, China. \\ *Corresponding author's email: sxsd456 [AT] sina.com
}

\begin{abstract}
Chemical composition of the essential oil from Qiancao roots was analyzed by gas chromatographymass spectrometry (GC-MS), and 43 components were identified in the paper. Mollugin (30.58\%) was found to be the major component, followed by 3-methyl-2-cyclopenten-1-one (10.55\%), 4-tert-butyl-2-phenyl-phenol (8.64\%) and eugenol (6.30\%). The antibacterial activity of essential oil against selected food-borne pathogens was evaluated. The results showed that the sensitivity to essential oil were different for different tested bacteria and the essential oil had a strong antibacterial activity against B. cereus with the lowest minimum inhibitory concentration (MIC) and minimum bactericide concentration (MBC) values of 0.0625 and $0.125 \mathrm{mg} / \mathrm{mL}$, respectively. In addition, microbial growth curve assay also showed that the essential oil had a significant effect on the growth rate of surviving B. cereus. The data of this study suggests that the essential oil from Qiancao roots has great potential for application as a natural antimicrobial agent to preserve food.
\end{abstract}

Keywords—Rubia cordifolia, chemical composition, antimicrobial activity, essential oil

\section{INTRODUCTION}

Food poisoning and spoilage caused by microorganisms results in enormous losses of foods, and has been of vital concern to public health (Sokmen et al., 2004; Diao et al., 2014). There have been many attempts by using synthetic chemicals to diminish microbial growth or inhibit microorganisms (Gao et al., 2011). However, consumers have become increasingly concerned about the adverse effects of synthetic antimicrobial chemicals and want safer materials for preventing and controlling pathogenic microorganisms in foods. Thus, development natural antibacterial compounds and novel antimicrobial agents are not only becoming critically important but also have attracted increasing interest of researchers (Ma et al., 2015).

Rubia cordifolia L., known as Qiancao in Chinese, is a well-known medicinal plant, belonging to the family Rubiaceae. In recent years, studies on Qiancao attracted more attention due to its potential biology efficacy and a number of the bioactive experiments on the roots extract have been reported from Qiancao (Liu et al., 2008; Divakar et al., 2010; Kaur et al., 2010; Lodia et al., 2012). However, to the best of our knowledge, little work has been reported on the chemical composition and antibacterial activity of essential oil from Qiancao on the growth of microorganisms. Hence, we have conducted the present study to explore the chemical composition and antibacterial activity of essential oil from Qiancao roots on several selected food-borne pathogens.

\section{MATERIALS AND METHODS}

\subsection{Plant material and chemicals}

The plant material was collected from Weinan, Shaanxi, China. The plant material was identified and duly authenticated by Dr. Ren Yi of Center for Advanced Studies in Botany of Shaanxi Normal University. A voucher specimen of the collected sample was deposited in the departmental herbarium. The main laboratory chemicals such as nalkanes $\mathrm{C}_{8}-\mathrm{C}_{22}$, nutrient agar (NA) and nutrient broth (NB) mediums were respectively purchased from Sigma (USA) and Beijing-Aoboxing Corporation (China). Other chemicals used were all of analytical grade.

\subsection{Microbial strains and culture}

In vitro antimicrobial activity of the essential oil was tested against seven kinds of microorganisms including four Gram-positive and there Gram-negative strains. Four Gram-positive strains were Staphylococcus albus ATCC 8799, 
Staphylococcus aureus ATCC 25923, Bacillus subtilis ATCC 6051 and Bacillus cereus A018. Three Gram-negative strains were Salmonella typhimurium ATCC 19430, Shigella dysenteriae CMCC (B) 51252 and Escherichia coli ATCC 25922. All tested strains were provided by the College of Engineering, Shanxi Normal University, and stored with liquid paraffin wax at $4{ }^{\circ} \mathrm{C}$.

\subsection{Extraction of the essential oil}

The $100 \mathrm{~g}$ of air-dried and roughly powered plant roots collected randomly were subjected to hydrodistillation for $4 \mathrm{~h}$ with a Clevenger-type apparatus in accordance with the Chinese Pharmacopoeia. The essential oil obtained on glass tube wall of essential oil receiver was collected, dried with anhydrous sodium sulfate and stored in a tightly closed dark vial at $4{ }^{\circ} \mathrm{C}$ until chemical composition analysis. The essential oil was obtained as a light yellow-green tabular crystal and had distinct sharp odour with a $0.12 \%$ yield $(\mathrm{w} / \mathrm{w})$.

\section{$2.4 G C$ and $G C$-MS analysis}

Gas chromatography equipped with flame ionization detection (GC-FID) and gas Chromatography-mass spectrometry (GC-MS) was adopted to analyze the essential oil from Qiancao roots. The fused silica capillary column $(30 \mathrm{~m} \times 0.25 \mathrm{~mm}$; film thickness $0.25 \mathrm{~mm}$ ) was Agilent Technology HP-5ms and temperature for injector and detector were maintained at $250{ }^{\circ} \mathrm{C}$. Oven temperature was programed as: $40-250{ }^{\circ} \mathrm{C}\left(2{ }^{\circ} \mathrm{C} / \mathrm{min}\right)$ and isotherm at $250{ }^{\circ} \mathrm{C}$ for 5 min. The carrier gas was nitrogen at a flow rate of $1.5 \mathrm{~mL} / \mathrm{min}$. Sample was dissolved in ethyl acetate at $2 \mathrm{mg} / \mathrm{mL}$; injection volume and split ratio was separately $2.0 \mu \mathrm{L}$ and 1:50. GC-MS was conducted with the equipment from SHIMADZU (Japan). A fused silica capillary SHIMADZU Rxi-5SilMS (5\% phenyl methyl siloxane) column (30 m×0.25 mm; film thickness $0.25 \mathrm{~mm}$ ) was used for the separation. The diluted oil sample $(2 \mathrm{mg} / \mathrm{mL}$ in ethyl acetate $)$ was injected into the column using a 1:50 split ratio. The operating conditions were the same as described above. Flow rate of carrier gas (Helium) was 1.30 $\mathrm{mL} / \mathrm{min}$ (constant flow). The mass spectrometer was operated in electron-impact ionization (EI) mode at 70eV. The scan condition was 50-350 amu/0.5 s per scan. The retention indices for identified volatile constituents were calculated using a homologous series of n-alkanes $\mathrm{C}_{8}-\mathrm{C}_{22}$. The essential oil constituents were verdicted by comparing their retention indices, mass spectra with publish data and National Institute of Standards and Technology mass spectra library data provided by the software of GC-MS system.

\subsection{Determination of antimicrobial activity}

The essential oil was dissolved in DMSO (Sigma) and sterilized by filtration through 0.22 mm Millipore filters. The antimicrobial activity of the essential oil was evaluated using the standardized filter paper disk diffusion method using $100 \mu \mathrm{L}$ of standardized inoculum containing $1 \times 10^{7}$ colony-forming units $(\mathrm{CFU}) / \mathrm{mL}$ of bacteria suspension poured. The diameter of inhibition zone (DIZ) was measured after $24 \mathrm{~h}$ of incubation at $37^{\circ} \mathrm{C}$. Tests were performed in triplicate.

\subsection{Determination of minimum inhibitory concentration (MIC) and minimum bactericidal concentration $(\mathrm{MBC})$}

The MIC values were determined with Resazurin microtitre-plate assay (REMA) by tube dilution method as described by Sarker et al. (Sarker et al., 2007). Plates were prepared under aseptic conditions. A sterile 96 well plate was labelled (Fig.1). Briefly, $5 \mathrm{~mL}$ of nutrient broth and $8 \mathrm{~mL}$ of resazurin indicator solution $(0.07 \mathrm{~g} / 100 \mathrm{~mL})$ were mixed together to get the mixture solution and then $130 \mu \mathrm{L}$ of the mixture solution was pipetted into to the tested wells of the plate. A volume of $10 \mu \mathrm{L}$ of the essential oil solutions $(8 \mathrm{mg} / \mathrm{mL}$, w/v in DMSO), the solvent of the essential oil (DMSO) as negative control and standard antibiotic Gentamicin $(0.0625 \mathrm{mg} / \mathrm{mL}$ in DMSO solution) as positive control was respectively pipetted into the first, the tenth and last column of the 96 well plates. At the same time, $10 \mu \mathrm{L}$ of two fold serial dilutions of the essential oil solutions were added to all other wells (from the second to the ninth column of well). Finally, $50 \mu \mathrm{L}$ of bacterial suspension of 7 tested strains $\left(1 \times 10^{7} \mathrm{cfu} / \mathrm{mL}\right)$ was added to each well except the eleventh column of well, which was used as sterile control (only with the mixture solution of the resazurin indicator and nutrient broth). Each plate was wrapped loosely with cling film to ensure that bacteria did not become dehydrated. The plates were prepared in triplicate, and placed in an incubator set at $37{ }^{\circ} \mathrm{C}$ for $18 \mathrm{~h}$. The color change was then assessed visually. The growth was indicated by color changes from purple to pink (or colorless). A change in color of growth control well to pink indicated the proper growth of the isolate and no change in color of sterile control well indicated absence of contaminants. The lowest concentration at which color change occurred was taken as the MIC value. The average of three values was determined and that was the MIC for the test material and bacterial strain.

The wells showing complete absence of growth in the MIC assay were identified and $10 \mu \mathrm{L}$ of each well were transferred to nutrient agar plates (Hussain et al., 2011). The agar plates were incubated with time-temperature profiles identical to that in disc diffusion assay. The well showing the complete absence of growth on agar plates was considered as the minimum bactericidal concentration (MBC). 


\subsection{Microbial growth curve assay}

The effect of essential oil on the growth of tested bacterial strain was evaluated with a slightly modified method (Diao et al., 2014). Briefly, logarithmic phase strain was diluted to $1 \times 10^{7} \mathrm{CFU} / \mathrm{mL}$ with nutrient broth. The essential oil was added to the cultures to keep the final concentrations of $0.5 \times, 1 \times$ and $2 \times \mathrm{MIC}$. The cultures were incubated in $\mathrm{NB}$ at $37{ }^{\circ} \mathrm{C}$ and $120 \mathrm{rpm}$. At selected time intervals, samples from test culture were taken and the absorbance at $595 \mathrm{~nm}$ was measured.

\subsection{Statistical analysis}

All experiments were conducted three times independently and the experimental data were expressed as mean \pm standard deviation. Analysis of variance and Duncan's multiple range tests were carried out to determine significant differences $(\mathrm{p}<0.05)$.

\section{RESULTS AND DISCUSSION}

\subsection{Chemical composition of the essential oil}

The essential oil from dried Qiancao roots was analyzed on GC and GC/MS. The identified compounds of the sample were listed in Table1. The 43 constituents were tentatively identified, representing $92.58 \%$ of all components in the essential oil. The mollugin (30.58\%), 3-methyl-2-Cyclopenten-1-one (10.55\%), 4-tert-butyl-2-phenyl-Phenol (8.64\%) and eugenol $(6.30 \%)$ were the major components of the oil. As regards the oils from Osaka studied by Miyazawa (Miyazawa et al., 2006), 62 components were once identified and the main components were mollugin (19.6\%), furomollugin (17.4\%), eugenol (12.7\%), (E)-anethole (10.6\%), and 4-tert-butyl-2-pheyl phenol (9.9\%) in the essential oil of Qiancao roots. However, in the past studies there was no relevant report on the identification of the substance existing in any natural essential oil. It's worth noting that another compositional feature of our sample is the occurrence of anthraquinones. As for anthraquinones, another important compound for Rubia species, they have been often used in food processing industries as well as in medicine for their biological activities (Han et al., 2001). To the best of our knowledge, this kind of compound is been reported for the first time as a constituent of the essential oil from Qiancao roots.

Table 1 Chemical composition of the essential oil from $R$. cordifolia roots

\begin{tabular}{|c|c|c|c|c|c|}
\hline Compound & $\mathrm{RI}^{\mathrm{a}}$ & $\begin{array}{c}\text { Peak area } \\
(\%)\end{array}$ & Compound & RI & Peak $\operatorname{area}(\%)$ \\
\hline 2-Cyclopenten-1-one, 3-methyl & 843 & 10.55 & Heneicosane & 2100 & 0.48 \\
\hline Benzaldehyde & 965 & 0.20 & Mollugin & 2424 & 30.58 \\
\hline Benzene acetaldehyde & 1046 & 0.18 & 9-octadecenoic acid, methyl ester & 2085 & 0.92 \\
\hline Borneol & 1168 & 0.22 & n- Eicosane & 2000 & 0.40 \\
\hline Menthol & 1178 & 2.25 & 9,10-anthracenedione, 2-methyl & 2069 & 1.74 \\
\hline 4-Terpineol & 1182 & 0.32 & Linoleic acid & 2125 & 0.10 \\
\hline (E)-cinnamaldehyde & 1278 & 0.92 & Anthracene & 2161 & 0.18 \\
\hline 2-undecanone & 1302 & 0.66 & 1-[4-(4-Acetylbenzyl)phenyl]ethanone & 2164 & 0.59 \\
\hline Dodecane, 2,6,11-trimethyl & 1320 & 0.21 & 2-t-butyl-4-(dimethylbenzyl) phenol & 2128 & 2.21 \\
\hline Eugenol & 1360 & 6.30 & Chalcone & 2251 & 0.21 \\
\hline Methyl eugenol & 1403 & 0.49 & 1-hydroxy-4-methylanthraquinone & 2290 & 3.68 \\
\hline Myristicin & 1526 & 0.51 & 9,10-anthracenedione, 2-droxymethyl & 2312 & 0.56 \\
\hline$\beta$-eudesmol & 1650 & 0.61 & $\begin{array}{l}\text { 1-Hydroxy-3-methoxy-6- } \\
\text { methylanthraquinone }\end{array}$ & 2479 & 4.32 \\
\hline $\begin{array}{l}\text { 2-(1,1-dimethylethyl)-3,4- } \\
\text { dihydro-1(2H)-naphthalenone }\end{array}$ & 1613 & 1.48 & 1,8,9-anthracenetriol & 2557 & 0.56 \\
\hline Heptadecane, 8-methyl & 1746 & 0.39 & Hexacosane & 2600 & 0.68 \\
\hline Hexahydrofarnesyl acetone & 1754 & 0.86 & Octacosane & 2800 & 0.21 \\
\hline Hexadecanoic acid, methyl ester & 1878 & 0.44 & 11-hydroxycannabinol & 2824 & 4.38 \\
\hline 4-tert-butyl-2-phenyl- phenol & 1915 & 8.64 & Ditridecyl ketone & 2841 & 0.30 \\
\hline Hexadecanoic acid & 1968 & 0.60 & Supraene & 2914 & 0.48 \\
\hline Hexadecanoic acid, ethyl ester & 1978 & 0.50 & 1-eicosanol & 3778 & 2.19 \\
\hline Ethanone & 1999 & 1.48 & Total & & 92.58 \\
\hline
\end{tabular}

${ }^{a}$ Retention indices relative to $\mathrm{C}_{8}-\mathrm{C}_{20}$ n-alkanes on the Rxi-5SilMS capillary column. 


\subsection{DIZ, MIC and MBC of the essential oil}

The DIZ, MIC, and MBC values of the essential oil from Qiancao roots are presented in Table 2. The results showed that the essential oil had a satisfactory antibacterial activity on all of the tested food-borne pathogens, including both Gram-positive and Gram-negative bacteria. The DIZ values for all tested bacterial strains were in the range of 10.3-18.3 $\mathrm{mm}$. The DIZ was the maximum value for $B$. cereus, followed by $S$. albus and $S$. dysenteriae, the lowest for $E$. coli. The MIC and MBC values for tested bacterial strains were in the range of $0.0625-0.25 \mathrm{mg} / \mathrm{mL}$ and $0.125-4.0 \mathrm{mg} / \mathrm{mL}$ (Figure S1), respectively. Of these bacteria, the essential oil performed both a minimum MIC of $0.0625 \mathrm{mg} / \mathrm{mL}$ and a minimum MBC of $0.125 \mathrm{mg} / \mathrm{mL}$ against $B$. cereus, which indicated it was the most effective bacterial inhibitor and bactericide against B. cereus. Nevertheless, the antibacterial activity of essential oil from Qiancao roots was far lower than that of Gentamicin.

Table 2 DIZ, MIC, and MBC of the essential oil from Qiancao roots.

\begin{tabular}{|c|c|c|c|c|c|c|}
\hline & \multicolumn{3}{|c|}{ Essential oil } & \multicolumn{3}{|c|}{ Gentamicin } \\
\hline & DIZ & MIC & $\mathrm{MBC}$ & DIZ & MIC & MBC \\
\hline \multicolumn{7}{|l|}{$\mathrm{G}^{+}$} \\
\hline S. aureus & $13.6 \pm 2.5$ & 0.125 & 0.5 & $29.0 \pm 0.8$ & $\leq 0.0625$ & $\leq 0.0625$ \\
\hline B. subtilis & $12.2 \pm 0.5$ & 0.25 & 0.5 & $19.5 \pm 0.9$ & $\leq 0.0625$ & $\leq 0.0625$ \\
\hline S. albus & $15.7 \pm 0.8$ & 0.0625 & 0.25 & $23.8 \pm 0.9$ & $\leq 0.0625$ & $\leq 0.0625$ \\
\hline B. cereus & $18.3 \pm 1.7$ & 0.0625 & 0.125 & $24.6 \pm 2.3$ & $\leq 0.0625$ & $\leq 0.0625$ \\
\hline \multicolumn{7}{|l|}{$\mathrm{G}^{-}$} \\
\hline S. typhimurium & $14.3 \pm 2.1$ & 0.125 & 0.25 & $21.6 \pm 0.7$ & $\leq 0.0625$ & $\leq 0.0625$ \\
\hline E. coli & $10.3 \pm 0.4$ & 1.0 & 4.0 & $24.0 \pm 0.6$ & $\leq 0.0625$ & $\leq 0.0625$ \\
\hline S. dysenteriae & $15.5 \pm 0.4$ & 0.125 & 0.125 & $24.8 \pm 0.8$ & $\leq 0.0625$ & $\leq 0.0625$ \\
\hline
\end{tabular}

Values represent means of three independent replicates \pm SD.

\subsection{Microbial growth curve assay}

To further confirm the antibacterial activity of essential oil against B. cereus, the time courses of B. cereus growth in the presence of different concentrations of essential oil were plotted. As shown in Figure 1, the growth of B. cereus was completely inhibited after $24 \mathrm{~h}$ of incubation at the concentrations of $1 \mathrm{MIC}$ and $2 \mathrm{MIC}$. On the other hand, $0.5 \mathrm{MIC}$ concentrations of essential oil hardly affected the growth of $S$. aureus but did increase the lag time in the growth curve. These findings demonstrated that the antibacterial effect of essential oil from Qiancao roots was in a dose-dependent manner and increased with the dose.

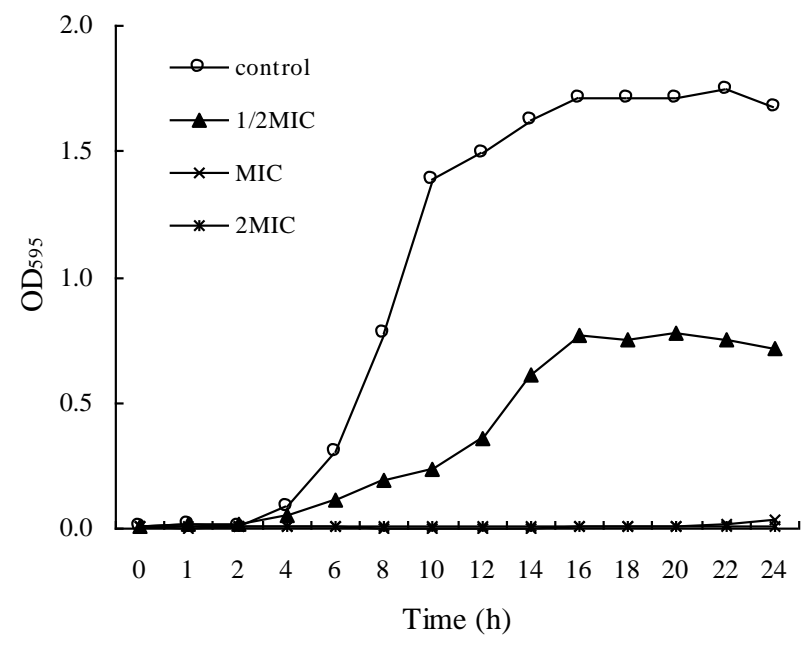

Figure 1: Effect of the essential oil from Qiancao roots on Bacillus cereus growth

\section{CONCLUSION}

Based on the present research, the essential oil from Qiancao roots, which was rich in mollugin and anthraquinones, possessed good antibacterial activity against selected food-borne pathogens in this study. The essential oil exhibited the 
strongest inhibitory effect against $B$. cereus, whereas the weakest effect was found against E. coli among all the tested microorganisms. The results of microbial growth curve indicated the treatment time and concentration of essential oil had great influences on antibacterial effects. In view of the present results, it is concluded that the essential oil from Qiancao roots has great potential for application as a natural antimicrobial agent to preserve food against the well-known causal agents of foodborne diseases and food spoilage. This is the first report on antimicrobial activity of the essential oil from Qiancao roots. Further research, particularly on the mode of action of main bioactive components in the essential oil from Qiancao roots, is still necessary.

\section{REFERENCES}

1. Diao W-R, Hu Q-P, Zhang H, Xu, J-G. 2014. Chemical composition, antibacterial activity and mechanism of action of essential oil from seeds of fennel (Foeniculum vulgare Mill.). Food Control 35: 109-116.

2. Divakar K, Pawar AT, Chandrasekhar SB, Dighe SB, Divakar G. 2010. Protective effect of the hydro-alcoholic extract of Rubia cordifolia roots against ethylene glycol induced urolithiasis in rats. Food and Chemical Toxicology 48: 1013-1018.

3. Gao CY, Tian CR, Lu YH, Xu JG, Luo JY, Guo XP. 2011. Essential oil composition and antimicrobial activity of Sphallerocarpus gracilis seeds against selected food-related bacteria. Food Control 22: 517-522.

4. Han Y-S, der Heijden RV, Verpoorte R. 2001. Biosynthesis of anthraquinones in cell cultures of Rubiaceae. Plant Cell Tissue and Organ Culture 67: 201-220.

5. Hussain AI, Anwar F, Nigam PS, Sarker SD, Moore JE, Rao JR, Mazumdar A. 2011. Antibacterial activity of some Lamiaceae essential oils using resazurin as an indicator of cell growth. LWT-Food Science and Technology 44: 1199-1206.

6. Kaur P, Chandel M, Kumar S, Kumar N, Singh B, Kaur S. 2010. Modulatory role of alizarin from Rubia cordifolia L. against genotoxicity of mutagens. Food and Chemical Toxicology 48: 320-325.

7. Liu R, Lu YB, Tian X, Pan Y. 2008. Simultaneous isolation and purification of mollugin and two anthraquinones from Rubia cordifolia by HSCCC. Chromatographia 28(1): 95-99.

8. Lodia S, Kansala L. 2012. Antioxident activity of Rubia cordifolia against lead toxicity. International Journal of Pharmaceutical Science and Reseach 3(7): 2224-2232.

9. Ma TT, Luo JY, Tian CR, Sun XY, Quan MP, Zheng CP, Kang LN, Zhan JC. 2011. Influence of technical processing units on chemical composition and antimicrobial activity of carrot (Daucus carrot L.) juice essential oil. Food Chemistry 170: 394-400.

10. Miyazawa M, Kawata J. 2006. Identification of the key aroma compounds in dried roots of Rubia cordifolia. Journal of Oleo Science 1(55): 37-39.

11. Sarker SD, Nahar L, Kumarasamy Y. 2007. Microtitre plate-based antibacterial assay incorporating resazurin as an indicator of cell growth, and its application in the in vitro antibacterial screening of phytochemicals. Methods 42: 321-324.

12. Sokmen A, Gulluce M, Akpulat HA, Daferera D, Tepe B, Polissiou M, Sokmen M, Sahin, F. 2004. The in vitro antimicrobial and antioxidant activities of the essential oils and methanol extracts of endemic Thymus spathulifolius. Food Control 15: 627-634. 